\title{
A direct method for evaluating arbitrary high-order singular curved boundary integrals
}

\author{
Xiaowei Gao, Weizhe Feng, Jinjun Zhao \& Miao Cui \\ State Key Laboratory of Structural Analysis for Industrial Equipment, \\ School of Aeronautics and Astronautics, \\ Dalian University of Technology, China
}

\begin{abstract}
In this paper, an efficient method for numerical evaluation of all kinds of singular curved boundary integrals from 2D/3D BEM analysis is proposed based on an operation technique on a projection line/plane. Firstly, geometry variables on a curved line or surface element are expressed by parameters on the projection line/plane, and then all singularities are analytically removed by expressing the non-singular part of the integration kernel as a power series in a local distance defined on the projection line/plane. Also, a set of important relationships computing derivatives of intrinsic coordinates with respect to local orthogonal coordinates is derived. A few examples are provided to demonstrate the correctness and the stability of the proposed method.

Keywords: boundary element method, super singular integral, projection plane, power series expansion, radial integration method (RIM).
\end{abstract}

\section{Introduction}

The efficient analysis of engineering problems using the boundary element method (BEM) requires accurate evaluation of various kinds of boundary integrals. When the source point is located on the element under consideration, some boundary integrals become singular. The treatment of these singular boundary integrals has been being an important topic for BEM researchers, and some methods have been proposed [1-8] for eliminating involved singularities. So far, the efficient methods treating singular curved line or surface integrals are the ones based on the operation over the intrinsic coordinate system [1-3].

Guiggiani et al. [2] proposed a general algorithm for evaluating hypersingular curved surface integrals based on expansion of geometry quantities as 
Laurent series in the parameter plane of intrinsic coordinates. This algorithm has been developed to evaluate super-singular boundary integrals by Karami and Derakhshan [3] and high-order singularities by Frangi and Guiggiani [4]. In a different way, Bonnet and Guiggiani [5] presented an algorithm for evaluating hyper-singular double integrals arising in 2D Galerkin BEM. Recently, Gao [1] proposed a method for handling super-singular boundary integrals with the order of singularities up to 6 by expanding the non-singular part of the integrand as power series in the intrinsic plane. The common point of these methods is that the parameter plane of intrinsic coordinates is used to set up the polar coordinate system [2-6] or to using RIM [1] to eliminate high-order singularities. The drawback of these methods is that a small contour of an inner neighbourhood around the source point, which is distorted in the parameter plane, needs to be reversed to express in terms of a same global size quantity. This limits the scale of eliminating singularity orders.

In this paper, a novel direct method for evaluating arbitrary high-order singular boundary integrals is proposed by projecting geometry quantities over a curved line for two-dimensional (2D) problems or a curved surface for threedimensional (3D) problems onto a tangential line or a fat plane to the center of the boundary element. Then, various singularities are removed analytically on the projection line/plane. In doing so, a set of important formulae for computing derivatives of intrinsic coordinates with respect to local orthogonal coordinates are derived, which provides a basis for expanding all geometry quantities as Taylor series in terms of the distance defined on the projection line/plane. Some 2D and 3D numerical examples are given, which show that very stable results can be achieved using the proposed method in the paper.

\section{Singular boundary integrals and boundary element discretization}

In analysis of engineering problems using BEM, the boundary of the problem is usually discretized into a series of boundary elements [1,9]. Singular integrals over an element can be classified into the following form [1]:

$$
I_{e}\left(\boldsymbol{x}^{p}\right)=\int_{\Gamma_{e}} \frac{\bar{f}\left(\boldsymbol{x}^{p}, \boldsymbol{x}\right)}{r^{\beta}\left(\boldsymbol{x}^{p}, \boldsymbol{x}\right)} d \Gamma(\boldsymbol{x})
$$

in which, $\boldsymbol{x}^{p}$ and $x$ represent the source and field points, respectively; $\Gamma_{e}$ is the boundary element under integration, which is a curved line or a curved surface for $2 \mathrm{D}$ or $3 \mathrm{D}$ problems, respectively; and $r$ is the distance between the source and field points and is defined as:

$$
r\left(\boldsymbol{x}^{p}, \boldsymbol{x}\right)=\left\|\boldsymbol{x}-\boldsymbol{x}^{p}\right\|=\sqrt{\sum_{i=1}^{\mathrm{D}}\left(x_{i}-x_{i}{ }^{p}\right)^{2}}
$$

where $D=2$ for $2 \mathrm{D}$ and $D=3$ for $3 \mathrm{D}$ problems. The term $\bar{f}\left(\boldsymbol{x}^{p}, \boldsymbol{x}\right)$ in eqn. (1) is a regular function and $\beta$ is the order of the singularity.

In $2 \mathrm{D}$ problems, the following types of singular line integrals are also frequently encountered: 


$$
J_{e}\left(\boldsymbol{x}^{p}\right)=\int_{\Gamma_{e}} \frac{\bar{f}\left(\boldsymbol{x}^{p}, \boldsymbol{x}\right) \ln \left[r\left(\boldsymbol{x}^{p}, \boldsymbol{x}\right)\right]}{r^{\beta}\left(\boldsymbol{x}^{p}, \boldsymbol{x}\right)} d \Gamma(\boldsymbol{x})
$$

In this paper, it is assumed that the integrals shown in eqns (1) and (3) always exists, that is, the integration results are finite values.

In order to numerically evaluate the boundary integrals shown in eqns (1) and (3), coordinates in an element can be expressed in terms of their nodal values as follows:

$$
x_{i}(\xi)=\sum_{\alpha=1}^{N_{\text {node }}} N_{\alpha}(\xi) x_{i}^{\alpha}
$$

where $N_{\text {node }}$ is the number of element nodes; $\xi$ represents the intrinsic coordinate and for a surface element it being understood that $(\xi)=\left(\xi_{1}, \xi_{2}\right) ; N_{\alpha}$ is the shape function of the $\alpha$-th node [1], and $x_{i}^{\alpha}$ is the $i$-th component of coordinates at the $\alpha$-th node.

\section{Expansions of geometry quantities on the projection line or plane}

In general, boundary elements are some curved lines or surfaces, so direct evaluating integrals over them is difficult. To solve this problem, we introduce a projection line for $2 \mathrm{D}$ or a projection plane for $3 \mathrm{D}$ problems, which is the tangential line/plane of the element to the origin of the intrinsic coordinate system. A local orthogonal coordinate system is established on the projection line/plane. Assuming that the direction cosine of the local coordinate axes with respective to the global one is $L_{i j}$, (its determination method can be found in references, i.e., in [9]), the coordinates transformation between the local and global systems can be performed using the following relationships:

$$
\begin{gathered}
x_{i}^{\prime}=L_{i j}\left(x_{j}-x_{j}^{o}\right) \\
x_{i}-x_{i}^{o}=L_{j i} x_{j}^{\prime}
\end{gathered}
$$

where the repeated subscripts represent summation, $x_{i}^{o}$ is the global coordinates of the origin of the local coordinate system, which is determined using eqn. (4) by setting $\xi=0$.

Making use of eqn. (5), one can project the original curved element onto the projection line or plane to form a straight or a flat projection element, and then all geometry quantities can be expressed in terms of variables defined on the projection line/plane. 


\subsection{Expansion of geometry quantities on the projection line for 2D problems}

In $2 \mathrm{D}$ problems, the boundary element is a curved line as shown as in fig. 1 . The local orthogonal coordinate system is denoted by $\left(x_{1}^{\prime}, x_{2}^{\prime}\right)$ or $\left(x^{\prime}, y^{\prime}\right)$ and the projection line is a line along the axis $x_{1}^{\prime}$.

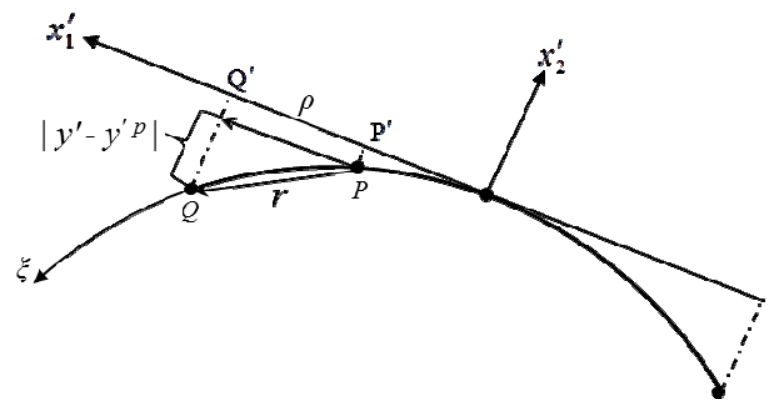

Figure 1: $\quad$ Variables defined on the projection line.

For a line, there is only one independent variable. Other geometry quantities on the curved line can be expressed in terms of this independent variable (here we choose the coordinate $x_{1}^{\prime}$ ). Thus, the coordinate $y^{\prime}$ (i.e., $x_{2}^{\prime}$ ) at the field point over the curved surface can also be expressed in terms of $x_{1}^{\prime}$. To do this, expanding $y^{\prime}$ as Taylor series about the origin of the local coordinate system as follows (truncated to quadratic terms):

$$
y^{\prime}=a_{1}\left(y^{\prime}\right) x_{1}^{\prime}+a_{2}\left(y^{\prime}\right) x_{1}^{\prime 2}
$$

where

$$
\begin{gathered}
a_{1}\left(y^{\prime}\right)=\frac{\partial y^{\prime}}{\partial x_{1}^{\prime}}=\frac{\partial y^{\prime}}{\partial \xi_{1}} \frac{\partial \xi_{1}}{\partial x_{1}^{\prime}} \\
a_{2}\left(y^{\prime}\right)=\frac{1}{2} \frac{\partial^{2} y^{\prime}}{\partial x_{1}^{\prime 2}}=\frac{1}{2}\left\{\frac{\partial^{2} y^{\prime}}{\partial \xi^{2}} \frac{\partial \xi}{\partial x_{1}^{\prime}} \frac{\partial \xi}{\partial x_{1}^{\prime}}+\frac{\partial y^{\prime}}{\partial \xi} \frac{\partial^{2} \xi}{\partial x_{1}^{\prime 2}}\right\}
\end{gathered}
$$

Through performing a differentiation for eqn. (4), we can obtain the following expressions for computing the first and second derivatives of the intrinsic coordinate with respect to the local coordinate.

$$
\begin{gathered}
\frac{\partial \xi}{\partial x_{1}^{\prime}}=\frac{1}{\left|m_{1}\right|} \\
\frac{\partial^{2} \xi}{\partial x_{1}^{\prime 2}}=-\frac{1}{\left|m_{1}\right|^{4}}\left(\frac{\partial x_{1}}{\partial \xi} \frac{\partial^{2} x_{1}}{\partial \xi^{2}}+\frac{\partial x_{2}}{\partial \xi} \frac{\partial^{2} x_{2}}{\partial \xi^{2}}\right)
\end{gathered}
$$

where 


$$
\left|\mathrm{m}_{1}\right|=\sqrt{\left(\frac{\partial x_{1}}{\partial \xi}\right)^{2}+\left(\frac{\partial x_{2}}{\partial \xi}\right)^{2}}
$$

In boundary integrals, usually a distance is used to formulate fundamental solutions. Therefore, it is convenient to define a local distance $\rho$ which is from the source point projection point $P^{\prime}$ to the field point projection point $Q^{\prime}$ (see fig. 1), i.e.,

$$
\rho=\left\|x_{1}^{\prime}-x_{1}^{\prime p}\right\|
$$

where $x_{1}^{\prime p}$ is the local coordinate of the source point $p$.

The local coordinate $x_{1}^{\prime}$ can be expressed in terms of the local distance $\rho$ using the following relationship $[10,11]$ :

$$
x_{1}^{\prime}=x_{1}^{\prime p}+\rho_{, 1} \rho
$$

where

$$
\rho_{, 1}=\frac{\partial \rho}{\partial x_{1}^{\prime}}=\frac{x_{1}^{\prime}-x_{1}^{\prime p}}{\rho}
$$

Thus, substituting eqn. (14) into eqn. (7), we obtain

$$
y^{\prime}=y^{\prime p}+q\left(y^{\prime}, \rho\right) \rho
$$

where

$$
\begin{gathered}
q\left(y^{\prime}, \rho\right)=q_{1}\left(y^{\prime}\right)+a_{2}\left(y^{\prime}\right) \rho \\
q_{1}\left(y^{\prime}\right)=\left[a_{1}\left(y^{\prime}\right)+2 a_{2}\left(y^{\prime}\right) x_{1}^{\prime p}\right] \rho_{, 1}
\end{gathered}
$$

In a similar manner, we can express the intrinsic coordinate in terms of the local distance as:

$$
\xi=\xi^{p}+q(\xi, \rho) \rho
$$

where $q(\xi, \rho)$ can be determined by replacing $y^{\prime}$ with $\xi$ in eqn. (17).

Referring to fig. 1, the global distance $r$ can be easily derived as

$$
r=\sqrt{\rho^{2}+\left(y^{\prime}-y^{\prime p}\right)^{2}}=g(\rho) \rho
$$

where

$$
g(\rho)=\sqrt{1+q^{2}\left(y^{\prime}, \rho\right)}
$$

Sometime, the global coordinates $x_{i}$ are also included in the integrand. Therefore, it is useful to derive the following expression from eqns (14) and (6) for $x_{i}$ :

$$
x_{i}=x_{i}^{p}+\left(b_{i}+c_{i} \rho\right) \rho
$$

where

$$
\begin{gathered}
b_{i}=L_{1 i} \rho_{, 1}+L_{2 i} q_{1}\left(y^{\prime}, \rho\right) \\
c_{i}=L_{2 i} a_{2}\left(y^{\prime}\right)
\end{gathered}
$$




\subsection{Expansion of geometry quantities on the projection plane for 3D problems}

In $3 \mathrm{D}$ problems, the boundary element is a curved surface as marked with $\mathrm{ABCD}$ in fig. 2. A local orthogonal coordinate system $\left(x^{\prime}, y^{\prime}, z^{\prime}\right)$, or written as $\left(x_{1}^{\prime}, x_{2}^{\prime}, x_{3}^{\prime}\right)$, is established on the projection plane with its origin being at the point $\left(\xi_{1}=0, \xi_{2}=0\right)$, in which the axes $x_{1}^{\prime}$ and $x_{2}^{\prime}$ are located within the plane with the axis $x_{1}^{\prime}$ being along $\xi_{1}$ direction and axis $x_{3}^{\prime}$ is along the outward normal direction of the element. Making use of eqn. (5), we can project the original curved surface element onto the projection plane, to form a flat projection element (marked with $A^{\prime} B^{\prime} C^{\prime} D^{\prime}$ ).

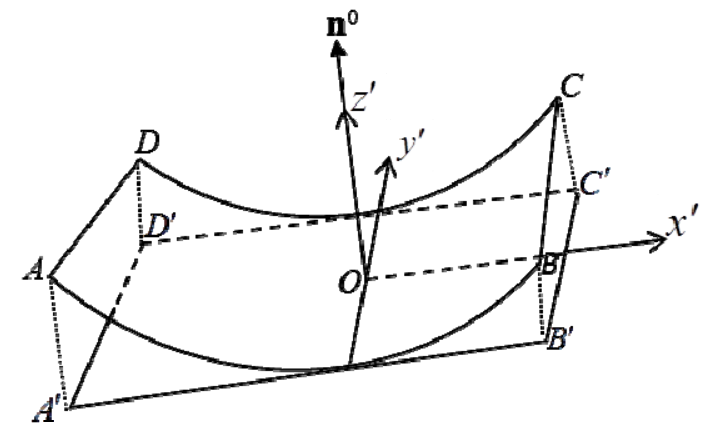

Figure 2: $\quad$ Boundary element projected onto projection plane.

In a plane, there are only two independent variables. We choose $x_{1}^{\prime}$ and $x_{2}^{\prime}$ as the independent variables, and $z^{\prime}$ (i.e., $x_{3}^{\prime}$ ) over the curved surface can be expressed in terms of $x_{1}^{\prime}$ and $x_{2}^{\prime}$. To do so, expanding $z^{\prime}$ as Taylor series about the origin of the local coordinate system as follows (truncated to quadratic terms):

$$
z^{\prime}=a_{I}\left(z^{\prime}\right) x_{I}^{\prime}+a_{I J}\left(z^{\prime}\right) x_{I}^{\prime} x_{J}^{\prime}
$$

in which, capital subscripts $I$ and $J$ take values from 1 to 2 , and

$$
\begin{gathered}
a_{I}\left(z^{\prime}\right)=\frac{\partial z^{\prime}}{\partial x_{I}^{\prime}}=\frac{\partial z^{\prime}}{\partial \xi_{K}} \frac{\partial \xi_{K}}{\partial x_{I}^{\prime}} \\
a_{I J}\left(z^{\prime}\right)=\frac{1}{2} \frac{\partial^{2} z^{\prime}}{\partial x_{I}^{\prime} \partial x_{J}^{\prime}}=\frac{1}{2}\left\{\frac{\partial^{2} z^{\prime}}{\partial \xi_{K} \partial \xi_{L}} \frac{\partial \xi_{K}}{\partial x_{I}^{\prime}} \frac{\partial \xi_{L}}{\partial x_{J}^{\prime}}+\frac{\partial z^{\prime}}{\partial \xi_{K}} \frac{\partial^{2} \xi_{K}}{\partial x_{I}^{\prime} \partial x_{J}^{\prime}}\right\}
\end{gathered}
$$

where $\partial z^{\prime} / \partial \xi_{K}$ can be calculated by nodal local coordinates using eqn. (4), expressions for $\partial \xi_{K} / \partial x_{I}^{\prime}$ have been given by Lachat in [12] and Lachat and Watson in [13], and expressions for $\frac{\partial^{2} \xi_{k}}{\partial x_{I}^{\prime} \partial x_{J}^{\prime}}$ are derived in this paper and listed in
the Appendix. 
Similar to $2 \mathrm{D}$ problems, the local distance $\rho$ projected from the global distance $r$ onto the projection plane and its derivatives $\rho_{, I}$ are introduced (fig. 3) as follows:

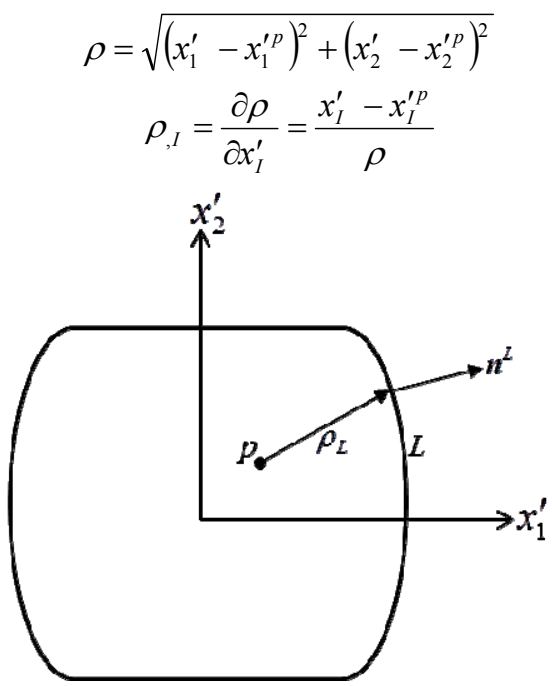

Figure 3: Quantities defined on the projection plane.

The local coordinates at the field point can be expressed in terms of a linear relationship on $\rho[10,11]$ as follows

$$
x_{I}^{\prime}=x_{I}^{\prime p}+\rho_{, I} \rho
$$

From eqn. (29) and fig. 3, it can be seen that $\rho_{, I}$ is a quantity depending on the angle between $\rho$ and axis $x_{1}^{\prime}$, being independent of $\rho$ itself.

Substituting eqn. (30) into eqn. (25) yields:

$$
z^{\prime}=z^{\prime p}+q\left(z^{\prime}, \rho\right) \rho
$$

where,

$$
q\left(z^{\prime}, \rho\right)=q_{1}\left(z^{\prime}\right)+q_{2}\left(z^{\prime}\right) \rho
$$

and,

$$
\begin{gathered}
q_{1}\left(z^{\prime}\right)=\left[a_{I}\left(z^{\prime}\right)+2 a_{I J}\left(z^{\prime}\right) x_{J}^{\prime p}\right] \rho_{, I} \\
q_{2}\left(z^{\prime}\right)=a_{I J}\left(z^{\prime}\right) \rho_{, I} \rho_{, J}
\end{gathered}
$$

In a similar manner to the $2 \mathrm{D}$ case, intrinsic and global coordinates can be expressed as:

$$
\begin{array}{r}
\xi_{K}=\xi_{K}^{p}+q\left(\xi_{K}, \rho\right) \rho \\
x_{i}=x_{i}^{p}+\left(b_{i}+c_{i} \rho\right) \rho
\end{array}
$$

where

$$
b_{i}=L_{J i} \rho_{, J}+L_{3 i} q_{1}\left(z^{\prime}\right)
$$




$$
c_{i}=L_{3 i} q_{2}\left(z^{\prime}\right)
$$

In eqn. (35), $q\left(\xi_{K}, \rho\right)$ can be determined by replacing $z^{\prime}$ with $\xi_{K}$ in eqn. (32).

Making use of the normalized orthogonal property of the coordinate transformation tensor $L_{i j}$, the expansion of $r$ can be derived using eqn. (36) as follows:

$$
r=\sqrt{\left(x_{i}-x_{i}^{p}\right)\left(x_{i}-x_{i}^{p}\right)}=g(\rho) \rho
$$

where,

$$
g(\rho)=\sqrt{1+q^{2}\left(z^{\prime}, \rho\right)}
$$

After expanding geometry quantities in terms of the local distance, we can treat the singular boundary integrals shown in eqns (1) and (3).

\section{Formulae for evaluating arbitrary singular boundary integrals}

\subsection{Formulae for evaluating singular curved line integrals}

In 2D problems, the differential relationship between the local distance and the real curved line can be written as :

$$
d \rho=d \Gamma n_{i}^{0} n_{i}
$$

where $n_{i}^{0}$ and $n_{i}$ are the outward normals to the origin of the local coordinate system and the field point, respectively.

Substituting eqns (40) and (38) into eqn. (1) leads to

$$
I_{e}\left(\boldsymbol{x}^{p}\right)=\lim _{\varepsilon \rightarrow 0} \int_{\varepsilon}^{\rho_{E}} \frac{\bar{F}(\rho)}{\rho^{\beta}} d \rho
$$

where $\rho_{E}$ is the distance from the projected point $P^{\prime}$ to one of the end of the projected line element, and $\bar{F}(\rho)$ is the regular part, that is,

$$
\bar{F}(\rho)=\frac{\bar{f}\left(\boldsymbol{x}^{p}, \boldsymbol{x}\right)}{g^{\beta}(\rho) n_{i}^{0} n_{i}}
$$

In order to integrate eqn. (41), the non-singular part $\bar{F}$ is expanded as a power series in $\rho$, such that

$$
\bar{F}(\rho)=\sum_{n=0}^{N} B^{(n)} \rho^{n}
$$

in which, $N$ is the order of the power series, usually taking a value between 2 and 7 depending the size of $\rho_{E}$; and $B^{(n)}$ are constants which are determined by collocating $N+1$ points over the integration region $\left(0, \rho_{E}\right)$. In the paper, $N+1$ equally spaced points are used, i.e., $\left(0, \rho_{1}, \cdots, \rho_{N}\right)$. The coefficient for the first point $(n=0)$ is $B^{(0)}=\bar{F}(0)$ and other coefficients can be solved using the following equation set: 


$$
[R]\{B\}=\{Y\}
$$

where, $[R]$ is a square matrix with the order of $N$ :

$\{B\}$ and $\{Y\}$ are vectors as follows:

$$
[R]=\left[\begin{array}{cccc}
1, & \rho_{1}, & \cdots & \rho_{1}^{N-1} \\
1, & \rho_{2}, & \cdots & \rho_{2}^{N-1} \\
\vdots & \vdots & \cdots & \vdots \\
1, & \rho_{N}, & \cdots & \rho_{N}^{N-1}
\end{array}\right]
$$

$$
\{B\}=\left\{\begin{array}{l}
B^{1} \\
B^{2} \\
\vdots \\
B^{N}
\end{array}\right\} \quad\{Y\}=\left\{\begin{array}{l}
{\left[\bar{F}\left(\rho_{1}\right)-B^{(0)}\right] / \rho_{1}} \\
{\left[\bar{F}\left(\rho_{2}\right)-B^{(0)}\right] / \rho_{2}} \\
\vdots \\
{\left[\bar{F}\left(\rho_{N}\right)-B^{(0)}\right] / \rho_{N}}
\end{array}\right\}
$$

Solving eqn. (44) for coefficient vector $\{B\}$ and then substituting eqn. (43) into (41) yield:

$$
I_{e}\left(\boldsymbol{x}^{p}\right)=\sum_{n=0}^{N} B^{(n)} \lim _{\varepsilon \rightarrow 0} \int_{\varepsilon}^{\rho_{E}} \rho^{n-\beta} d \rho=\sum_{n=0}^{N} B^{(n)} E_{n}
$$

where,

$$
E_{n}= \begin{cases}\frac{1}{n+1-\beta}\left(\rho_{E}^{n+1-\beta}-\lim _{\varepsilon \rightarrow 0} \varepsilon^{n+1-\beta}\right), & n \neq \beta-1 \\ \ln \rho_{E}-\lim _{\varepsilon \rightarrow 0} \ln \varepsilon, & n=\beta-1\end{cases}
$$

For a physical problem, the integral should exist. This means that the terms involving $\varepsilon$ in eqn. (48) should be eliminated after considering the contributions of the all adjacent elements around the source point or should be cancelled out by free terms [1-5]. Thus, we obtain:

$$
E_{n}=\left\{\begin{array}{lc}
\frac{-1}{\beta-n-1} \cdot \frac{1}{\rho_{E}^{\beta-n-1}}, & 0 \leq n \leq \beta-2 \\
\ln \rho_{E}, & n=\beta-1 \\
\frac{1}{n+1-\beta} \cdot \rho_{E}^{n+1-\beta}, & n>\beta-1
\end{array}\right.
$$

In a similar way, substituting eqns (40) and (38) into eqn. (3), it follows that

$$
J_{e}\left(\boldsymbol{x}^{p}\right)=\lim _{\varepsilon \rightarrow 0} \int_{\varepsilon}^{\rho_{E}} \frac{\bar{F}(\rho) \ln \rho}{\rho^{\beta}} d \rho+\lim _{\varepsilon \rightarrow 0} \int_{\varepsilon}^{\rho_{E}} \frac{\bar{F}^{\prime}(\rho)}{\rho^{\beta}} d \rho
$$

In which, the regular parts are:

$$
\begin{gathered}
\bar{F}(\rho)=\frac{\bar{f}\left(\boldsymbol{x}^{p}, \boldsymbol{x}\right)}{g^{\beta}(\rho) n_{i}^{0} n_{i}} \\
\bar{F}^{\prime}(\rho)=\frac{\bar{f}\left(\boldsymbol{x}^{p}, \boldsymbol{x}\right) \ln [g(\rho)]}{g^{\beta}(\rho) n_{i}^{0} n_{i}}
\end{gathered}
$$


In above equations, $\bar{F}$ is determined by eqn. (43), and $\bar{F}^{\prime}$ is expanded as the power series in $\rho$ as follows:

$$
\bar{F}^{\prime}(\rho)=\sum_{n=0}^{N} C^{(n)} \rho^{n}
$$

where coefficients $C^{(n)}$ can be determined using equations similar to eqns (44)-(46).

Through a similar treatment to eqn. (47), the following equation can be obtained from eqn. (50).

$$
J_{e}\left(\boldsymbol{x}^{p}\right)=\sum_{n=0}^{N} C^{(n)} E_{n}+\sum_{n=0}^{N} B^{(n)} H_{n}
$$

where $E_{n}$ is the same as eqn. (49), and $H_{n}$ is given below:

$$
H_{n}=\left\{\begin{array}{lc}
\frac{-\left\{(\beta-n-1) \ln \rho_{E}+1\right\}}{(\beta-n-1)^{2} \rho_{E}^{\beta-n-1}} & 0 \leq n \leq \beta-2 \\
\frac{1}{2} \ln ^{2} \rho_{E} & n=\beta-1 \\
\frac{(n+1-\beta) \ln \rho_{E}-1}{(n+1-\beta)^{2}} \rho_{E}^{n+1-\beta} & n>\beta-1
\end{array}\right.
$$

Equations (47) and (54) together with eqns (49) and (55) can be used to evaluate arbitrary high order of singular line integrals. Since $\rho_{E}$ cannot be zero, no singularities exist in the above equations.

\subsection{Formulae for evaluating singular curved surface integrals}

In $3 \mathrm{D}$ problems, the relationship between the differential areas over the projection plane and the real surface can be written as:

$$
d A=d \Gamma \cos \phi=d \Gamma n_{i}^{0} n_{i}
$$

in which, $A$ is the area of the projection plane, $n_{i}^{0}$ and $n_{i}$ are the outward normals to the origin of the local coordinate system and the field point, and $\phi$ is the angle between them.

Substituting eqns (38) and (56) into eqn. (1), the surface integral can be expressed in terms of an integral over the projection plane as follows:

$$
I\left(\boldsymbol{x}^{p}\right)=\int_{A} \frac{\bar{f}\left(\boldsymbol{x}^{p}, \boldsymbol{x}\right)}{g^{\beta}(\rho) \rho^{\beta} n_{i}^{0} n_{i}} d A(\boldsymbol{x})
$$

Employing the Radial Integration Method (RIM) [10, 11], the above integral over the projection plane can be transformed into a closed line integral over the contour of the projection element (see fig. 3):

$$
I\left(\boldsymbol{x}^{p}\right)=\int_{L} \frac{1}{\rho} \frac{\partial \rho}{\partial n^{L}} F d L
$$


in which, $\partial \rho / \partial n^{L}=\rho_{, I} n_{I}^{L}$ with $n_{I}^{L}$ being the outward normal to the contour line $L$ (fig. 3), and $\mathrm{F}$ is the radial integral on the projection plane and can be written as:

$$
F=\lim _{\varepsilon \rightarrow 0} \int_{\varepsilon}^{\rho_{L}} \frac{\bar{F}(\rho)}{\rho^{\beta-1}} d \rho
$$

where $\rho_{L}$ is the distance from the source point to the integration point on the contour line $L$, and $\bar{F}(\rho)$ has the same form as shown in eqn. (42).

Through a similar derivation process to the $2 \mathrm{D}$ problems, eqn. (59) can be computed using the following expression:

$$
F=\sum_{n=0}^{N} B^{(n)} E_{n}
$$

where the coefficient $B^{(n)}$ is determined using eqns (44)-(46), and

$$
E_{n}=\left\{\begin{array}{lc}
\frac{1}{n-\alpha+1} \frac{1}{\rho_{L}^{\alpha-n-1},} & 0 \leq n \leq \alpha-2 \\
\ln \rho_{L}, & n=\alpha-1 \\
\frac{1}{n-\alpha+1} \rho_{L}^{n-\alpha+1}, & n>\alpha-1
\end{array}\right.
$$

in which, $\alpha=\beta-1$.

Since $\rho_{L}$ is the distance from the source point $p$ to the contour line $L$ (see fig. 3), $\rho_{L}$ is not zero when the source point $p$ is located interior of the element. Otherwise, when $p$ is located on a side of $L$, the value of $\partial \rho / \partial n^{L}$ on the current element has the same size but opposite sign to that on the adjacent element, and therefore they cancel out each other. This means that the sides where $p$ is located on need not to be considered at all. Thus, arbitrary order of singular surface integrals can be evaluated using eqns (58), (60) and (61) without singularities.

\section{Numerical examples}

Based on the formulae derived in this paper, a computer code, SIETPEM (Singular Integral Evaluation based on Tangential Plane Expansion Method), has been developed, and a number of line and surface integrals have been evaluated numerically. Some of them are analyzed below.

\subsection{A singular integral over a parabolic curve}

The first example to be exhibited is a line integral with the form $J=\int_{\Gamma} \ln (r) d \Gamma$. The integration line is a parabolic curve as shown in fig. 4 with coordinates of three typical points being $1(0.0,0.0), 2(2.0,0.0), 3(1.0,0.5)$. The computational point (source point) is at 3 . The parabolic curve is taken as one $2 \mathrm{D}$ quadratic boundary element. This integral can be accurately evaluated using the Gauss quadrature rule for logarithmic function. The result is $J=-2.005257$. Table 1 
lists the computational results using the current method for different order of power series. It shows that the formulae derived in the paper is correct and can give very stable results.

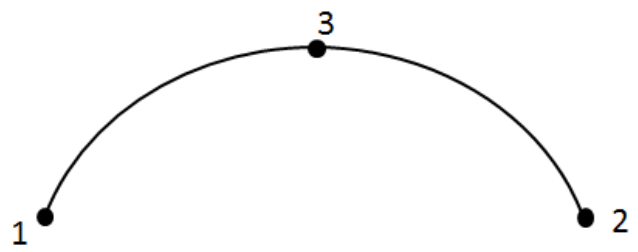

Figure 4: A parabolic curve.

Table 1: Computed results for various values of $\mathrm{N}$ (accurate result is $\mathrm{J}=-2.005257$ ).

\begin{tabular}{cccccc}
\hline$N$ & 3 & 4 & 5 & 7 & 9 \\
\hline$J$ & -2.004977 & -2.005137 & -2.005245 & -2.005258 & -2.005257 \\
\hline
\end{tabular}

\subsection{Singular integral over a $90^{\circ}$ cylinder surface}

The second example is adopted from Ref. [2] to examine the perforce of the proposed method in handling singular integrals over a highly curved surface. The boundary element considered is a cylinder surface with radius 1 and the rotational angle is $90^{\circ}$ (see fig. 5). The boundary integral is as follows:

$$
I\left(x^{p}\right)=\int_{\Gamma} \frac{-1}{4 \pi r^{\beta}}\left[3 r_{3} \frac{\partial r}{\partial n}-n_{3}\right] d \Gamma
$$

Three source points $a, b$ and $c$, are located at positions with intrinsic coordinates of $(0,0),(0.66,0)$ and $(0.66,0.66)$, respectively. Guiggiani et al. [2] computed this example in for the case of $\beta=3$. Table 2 lists the results from both methods, which shows that the current results are in good agreement with those from [2].

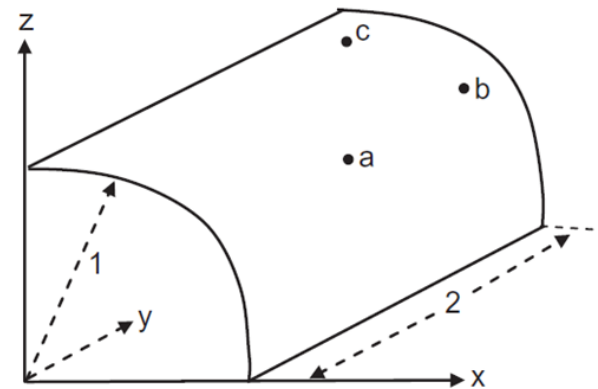

Figure 5: $\quad 90^{\circ}$ curved element. 
Table 2: $\quad$ Computed results for three points $a, b$ and $c(\beta=3)$.

\begin{tabular}{llll}
\hline & Point $a$ & Point $b$ & Point $c$ \\
\hline Ref. [2] & -0.343807 & -0.497119 & -0.876365 \\
Current & -0.343808 & -0.497095 & -0.876863 \\
\hline
\end{tabular}

\subsection{Singular integral over a $45^{\circ}$ spherical surface}

The third example is aimed to examine the super singular integral $I\left(x^{p}\right)=-\int_{\Gamma}\left[3 r_{, 3} \partial r / \partial n-n_{3}\right] /\left(4 \pi r^{\beta}\right) d \Gamma$ over a spherical surface with radius 1 . The element is formed by rotating a vertical circular arc with the height of $H=2 h=0.5$ by $45^{\circ}$ about the y-axis. Fig. 6 shows the 8 -noded spherical element.

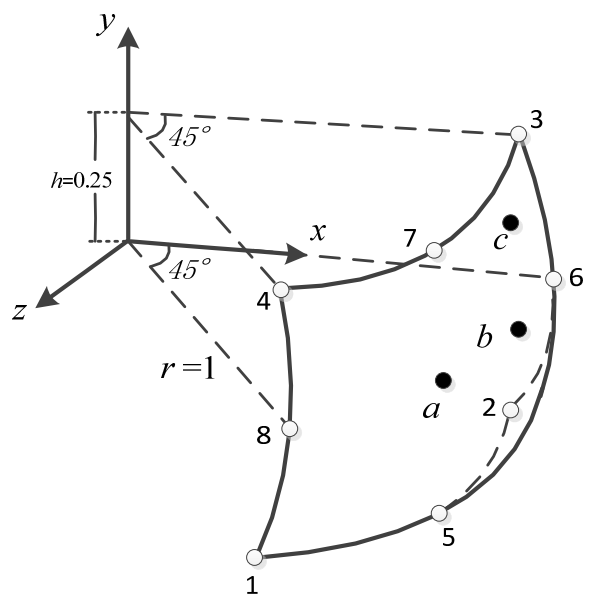

Figure 6: A spherical element with $45^{\circ}$ rotation angles.

Three source points $a, b$ and $c$ (see fig. 6$)$ with intrinsic coordinates $(0,0)$, $(0.5,0)$ and $(0.5,0.5)$, respectively, are computed. Tables 3 gives the computational results for the singularity order $\beta=3$ and Table 4 investigates the stability of the computational results to the order of the power series when treating the high-order singularity $\beta=6$. Comparison to results obtained using the method in [1] shows that two methods give relatively close results, but the current method is more stable than the method based on the treatment in the intrinsic coordinate plane presented in [1].

Table 3: $\quad$ Results for $\beta=3$.

\begin{tabular}{llll}
\hline & Point $a$ & Point $b$ & Point $c$ \\
\hline Current & -0.561422254 & -0.385803354 & -0.459634689 \\
Ref. [1] & -0.560328957 & -0.377519079 & -0.453400168 \\
\hline
\end{tabular}


Table 4: $\quad$ Results for different orders of power series at point $\mathrm{b}$ with $\beta=6$.

\begin{tabular}{lcccccc}
\hline$N$ & 2 & 3 & 4 & 5 & 6 & 7 \\
\hline Current & -6.926340 & -6.926340 & -6.926340 & -6.926083 & -6.927782 & -6.91993 \\
Ref.[1] & -6.668087 & -6.668087 & -6.668087 & -7.435129 & -10.60427 & -19.0199 \\
\hline
\end{tabular}

\section{Concluding remarks}

A new direct method for evaluating singular line and surface integrals has been presented in the paper. The derived formulae can handle arbitrary high order of singularities. The derived new set of formulae for computing derivatives of the intrinsic coordinates with respect to local orthogonal coordinates can provide a basis for expanding complicated geometry quantities as the Taylor series on the projection line or plane. The idea of expanding geometries as the Taylor series at the center of the element, rather than at the source point, can guarantee the high order of the computational accuracy.

\section{Acknowledgement}

The authors gratefully acknowledge the National Natural Science Foundation of China (11172055, 51206014, 11202045).

\section{Appendix: the second derivatives of intrinsic coordinates with respect to local orthogonal coordinates on a surface element}

$$
\begin{gathered}
\frac{\partial^{2} \xi_{1}}{\partial x_{1}^{\prime} \partial x_{1}^{\prime}}=-\frac{1}{\left|m_{1}\right|^{4}} \cdot\left(\frac{\partial x_{1}}{\partial \xi_{1}} \cdot \frac{\partial^{2} x_{1}}{\partial \xi_{1}^{2}}+\frac{\partial x_{2}}{\partial \xi_{1}} \cdot \frac{\partial^{2} x_{2}}{\partial \xi_{1}^{2}}+\frac{\partial x_{3}}{\partial \xi_{1}} \cdot \frac{\partial^{2} x_{3}}{\partial \xi_{1}^{2}}\right) \\
\frac{\partial^{2} \xi_{1}}{\partial x_{1}^{\prime} \partial x_{2}^{\prime}}=\frac{\cos \theta}{\left|m_{1}\right|^{4} \cdot \sin \theta} \cdot\left(\frac{\partial x_{1}}{\partial \xi_{1}} \cdot \frac{\partial^{2} x_{1}}{\partial \xi_{1}^{2}}+\frac{\partial x_{2}}{\partial \xi_{1}} \cdot \frac{\partial^{2} x_{2}}{\partial \xi_{1}^{2}}+\frac{\partial x_{3}}{\partial \xi_{1}} \cdot \frac{\partial^{2} x_{3}}{\partial \xi_{1}^{2}}\right) \\
-\frac{1}{\left|m_{1}\right|^{3} \cdot\left|m_{2}\right| \cdot \sin \theta} \cdot\left(\frac{\partial x_{1}}{\partial \xi_{1}} \cdot \frac{\partial^{2} x_{1}}{\partial \xi_{1} \partial \xi_{2}}+\frac{\partial x_{2}}{\partial \xi_{1}} \cdot \frac{\partial^{2} x_{2}}{\partial \xi_{1} \partial \xi_{2}}+\frac{\partial x_{3}}{\partial \xi_{1}} \cdot \frac{\partial^{2} x_{3}}{\partial \xi_{1} \partial \xi_{2}}\right) \\
\frac{\partial^{2} \xi_{1}}{\partial x_{2}^{\prime} \partial x_{1}^{\prime}}=\left(\frac{-\cos \theta}{\sin \theta}\right) \cdot\left[-\frac{1}{\left|m_{1}\right|^{4}} \cdot\left(\frac{\partial x_{1}}{\partial \xi_{1}} \cdot \frac{\partial^{2} x_{1}}{\partial \xi_{1}^{2}}+\frac{\partial x_{2}}{\partial \xi_{1}} \cdot \frac{\partial^{2} x_{2}}{\partial \xi_{1}^{2}}+\frac{\partial x_{3}}{\partial \xi_{1}} \cdot \frac{\partial^{2} x_{3}}{\partial \xi_{1}^{2}}\right)\right] \\
+\frac{1}{\left|m_{1}\right|^{2}} \cdot\left[\frac{1}{\sin \theta} \cdot \frac{\partial}{\partial \xi_{1}}(-\cos \theta)+(-\cos \theta) \cdot \frac{\partial}{\partial \xi_{1}}\left(\frac{1}{\sin \theta}\right)\right]
\end{gathered}
$$




$$
\begin{aligned}
\frac{\partial^{2} \xi_{1}}{\partial x_{2}^{\prime} \partial x_{2}^{\prime}} & =\left(\frac{\cos ^{2} \theta}{\sin ^{2} \theta}\right) \cdot\left[-\frac{1}{\left|m_{1}\right|^{4}} \cdot\left(\frac{\partial x_{1}}{\partial \xi_{1}} \cdot \frac{\partial^{2} x_{1}}{\partial \xi_{1}^{2}}+\frac{\partial x_{2}}{\partial \xi_{1}} \cdot \frac{\partial^{2} x_{2}}{\partial \xi_{1}^{2}}+\frac{\partial x_{3}}{\partial \xi_{1}} \cdot \frac{\partial^{2} x_{3}}{\partial \xi_{1}^{2}}\right)\right] \\
& -\frac{\cos \theta}{\sin \theta} \frac{1}{\left|m_{1}\right|^{2}} \cdot\left[\frac{1}{\sin \theta} \cdot \frac{\partial}{\partial \xi_{1}}(-\cos \theta)+(-\cos \theta) \cdot \frac{\partial}{\partial \xi_{1}}\left(\frac{1}{\sin \theta}\right)\right] \\
& +\left(\frac{-\cos \theta}{\left|m_{2}\right| \sin ^{2} \theta}\right) \cdot \frac{\partial}{\partial \xi_{2}}\left(\frac{1}{\left|m_{1}\right|}\right)+\frac{1}{\left|m_{1}\right|\left|m_{2}\right| \sin \theta} \\
& \cdot\left[\frac{1}{\sin \theta} \cdot \frac{\partial}{\partial \xi_{2}}(-\cos \theta)+(-\cos \theta) \cdot \frac{\partial}{\partial \xi_{2}}\left(\frac{1}{\sin \theta}\right)\right] \\
& \frac{\partial^{2} \xi_{2}}{\partial x_{2}^{\prime} \partial x_{1}^{\prime}}=\frac{1}{\left|m_{1}\right|} \frac{1}{\sin \theta} \frac{\partial}{\partial \xi_{1}}\left(\frac{1}{\left|m_{2}\right|}\right)+\frac{1}{\left|m_{1}\right|\left|m_{2}\right|} \frac{\partial}{\partial \xi_{1}}\left(\frac{1}{\sin \theta}\right) \\
\frac{\partial^{2} \xi_{2}}{\partial x_{2}^{\prime} \partial x_{2}^{\prime}} & =\frac{1}{\left|m_{1}\right|} \frac{-\cos \theta}{\sin ^{2} \theta} \frac{\partial}{\partial \xi_{1}}\left(\frac{1}{\left|m_{2}\right|}\right)+\frac{1}{\left|m_{1}\right|\left|m_{2}\right|} \cdot \frac{-\cos \theta}{\sin \theta} \frac{\partial}{\partial \xi_{1}}\left(\frac{1}{\sin \theta}\right) \\
& +\frac{1}{\left|m_{2}\right| \sin ^{2} \theta} \frac{\partial}{\partial \xi_{2}}\left(\frac{1}{\left|m_{2}\right|}\right)+\frac{1}{\left|m_{2}\right|^{2} \sin \theta} \frac{\partial}{\partial \xi_{2}}\left(\frac{1}{\sin \theta}\right)
\end{aligned}
$$

where,

$$
\begin{gathered}
\left|\mathrm{m}_{\mathrm{K}}\right|=\sqrt{\left(\frac{\partial x_{1}}{\partial \xi_{k}}\right)^{2}+\left(\frac{\partial x_{2}}{\partial \xi_{k}}\right)^{2}+\left(\frac{\partial x_{3}}{\partial \xi_{k}}\right)^{2}} \\
\cos \theta=\frac{1}{\left|m_{1}\right|\left|m_{2}\right|} \frac{\partial x_{i}}{\partial \xi_{1}} \frac{\partial x_{i}}{\partial \xi_{2}} \\
\frac{\partial}{\partial \xi_{1}}(\cos \theta)=\left[\frac{1}{\left|m_{2}\right|} \frac{\partial}{\partial \xi_{1}}\left(\frac{1}{\left|m_{1}\right|}\right)+\frac{1}{\left|m_{1}\right|} \frac{\partial}{\partial \xi_{1}}\left(\frac{1}{\left|m_{2}\right|}\right)\right] \cdot\left(\frac{\partial x_{1}}{\partial \xi_{1}} \cdot \frac{\partial x_{1}}{\partial \xi_{2}}+\frac{\partial x_{2}}{\partial \xi_{1}} \cdot \frac{\partial x_{2}}{\partial \xi_{2}}\right)+ \\
\frac{1}{\left|m_{1}\right|\left|m_{2}\right|}\left[\frac{\partial^{2} x_{1}}{\partial \xi_{1}^{2}} \cdot \frac{\partial x_{1}}{\partial \xi_{2}}+\frac{\partial x_{1}}{\partial \xi_{1}} \cdot \frac{\partial^{2} x_{1}}{\partial \xi_{1} \partial \xi_{2}}+\frac{\partial^{2} x_{2}}{\partial \xi_{1}^{2}} \cdot \frac{\partial x_{2}}{\partial \xi_{2}}+\frac{\partial x_{2}}{\partial \xi_{1}} \cdot \frac{\partial^{2} x_{2}}{\partial \xi_{1} \partial \xi_{2}}\right] \\
\frac{\partial}{\partial \xi_{1}}\left(\frac{1}{\sin \theta}\right)=\frac{1}{\sin ^{2} \theta} \cdot \frac{1}{2 \sqrt{1-\cos ^{2} \theta}} \cdot[2 \cos \theta] \cdot \frac{\partial}{\partial \xi_{1}}(\cos \theta) \\
\left.\frac{1}{\partial \xi_{2}}\left[\frac{1}{\left|m_{2}\right|} \frac{\partial}{\partial \xi_{2}}\left(\frac{1}{\left|m_{1}\right|}\right)+\frac{1}{\left|m_{1}\right|} \frac{\partial}{\partial \xi_{2}}\left(\frac{\partial^{2} x_{1}}{\left|m_{2}\right|}\right)\right] \cdot\left(\frac{\partial x_{1}}{\partial \xi_{1} \mid} \cdot \frac{\partial x_{1}}{\partial \xi_{2}}+\frac{\partial x_{2}}{\partial \xi_{1}} \cdot \frac{\partial x_{2}}{\partial \xi_{2}}\right)+\frac{\partial^{2} x_{1}}{\partial \xi_{2}^{2}}+\frac{\partial^{2} x_{2}}{\partial \xi_{1} \partial \xi_{2}} \cdot \frac{\partial x_{2}}{\partial \xi_{2}}+\frac{\partial x_{2}}{\partial \xi_{1}} \cdot \frac{\partial^{2} x_{2}}{\partial \xi_{2}^{2}}\right] \\
\frac{\partial}{\partial \xi_{2}}\left(\frac{1}{\sin \theta}\right)=\frac{1}{\sin ^{2} \theta} \cdot \frac{1}{2 \sqrt{1-\cos ^{2} \theta} \cdot[2 \cos \theta] \cdot \frac{\partial}{\partial \xi_{2}}(\cos \theta)}
\end{gathered}
$$




$$
\begin{gathered}
\frac{\partial}{\partial \xi_{1}}\left(\frac{1}{\left|m_{2}\right|}\right)=-\frac{1}{\left|m_{2}\right|^{3}} \cdot\left(\frac{\partial x_{1}}{\partial \xi_{2}} \cdot \frac{\partial^{2} x_{1}}{\partial \xi_{1} \partial \xi_{2}}+\frac{\partial x_{2}}{\partial \xi_{2}} \cdot \frac{\partial^{2} x_{2}}{\partial \xi_{1} \partial \xi_{2}}+\frac{\partial x_{3}}{\partial \xi_{2}} \cdot \frac{\partial^{2} x_{3}}{\partial \xi_{1} \partial \xi_{2}}\right) \\
\frac{\partial}{\partial \xi_{2}}\left(\frac{1}{\left|m_{2}\right|}\right)=-\frac{1}{\left|m_{2}\right|^{3}} \cdot\left(\frac{\partial x_{1}}{\partial \xi_{2}} \cdot \frac{\partial^{2} x_{1}}{\partial \xi_{2}^{2}}+\frac{\partial x_{2}}{\partial \xi_{2}} \cdot \frac{\partial^{2} x_{2}}{\partial \xi_{2}^{2}}+\frac{\partial x_{3}}{\partial \xi_{2}} \cdot \frac{\partial^{2} x_{3}}{\partial \xi_{2}^{2}}\right) \\
\frac{\partial}{\partial \xi_{1}}\left(\frac{1}{\left|m_{1}\right|}\right)=-\frac{1}{\left|m_{1}\right|^{3}} \cdot\left(\frac{\partial x_{1}}{\partial \xi_{1}} \cdot \frac{\partial^{2} x_{1}}{\partial \xi_{1}^{2}}+\frac{\partial x_{2}}{\partial \xi_{1}} \cdot \frac{\partial^{2} x_{2}}{\partial \xi_{1}^{2}}+\frac{\partial x_{3}}{\partial \xi_{1}} \cdot \frac{\partial^{2} x_{3}}{\partial \xi_{1}^{2}}\right) \\
\frac{\partial}{\partial \xi_{2}}\left(\frac{1}{\left|m_{1}\right|}\right)=-\frac{1}{\left|m_{1}\right|^{3}} \cdot\left(\frac{\partial x_{1}}{\partial \xi_{1}} \cdot \frac{\partial^{2} x_{1}}{\partial \xi_{1} \partial \xi_{2}}+\frac{\partial x_{2}}{\partial \xi_{1}} \cdot \frac{\partial^{2} x_{2}}{\partial \xi_{1} \partial \xi_{2}}+\frac{\partial x_{3}}{\partial \xi_{1}} \cdot \frac{\partial^{2} x_{3}}{\partial \xi_{1} \partial \xi_{2}}\right)
\end{gathered}
$$

\section{References}

[1] Gao, X.W., An effective method for numerical evaluation of general 2D and 3D high order singular boundary integrals, Comput Methods Appl. Mech. Engrg 199, pp. 2856-2864, 2010.

[2] Guiggiani, M., Krishnasamy, G., Rudolphi, T.J., Rizzo, F.J., A general algorithm for the numerical solution of hypersingular boundary integral equations, J Appl Mech, 59, pp. 604-14, 2010.

[3] Karami, G., Derakhshan, D., An efficient method to evaluate hypersingular and supersingular integrals in boundary integral equations analysis, Engineering Analysis with Boundary Element, 23, pp. 317-326,2010.

[4] Frangi, A., Guiggiani, M., A direct approach for boundary integral equations with high-order singularities, International Journal for Numerical Methods in Engineering, 49, pp. 871-898, 2010.

[5] Bonnet, M., Guiggiani, M., Direct evaluation of double singular integrals and new free terms in 2D (symmetric) Galerkin BEM, Computer Meth. Applied Mech and Engineering, 192, pp. 2565-2596, 2003.

[6] Huber, O., Lang, A. and Kuhn, G., Evaluation of the stress tensor in 3D elastostatics by direct solving of hypersingular integrals, Computational Mechanics, 12, pp. 39-50, 1993.

[7] Mukherjee, S., Mukherjee, Y.X., The hypersingular boundary contour method for three-dimensional linear elasticity. ASME Journal of Applied Mechanics, 65, pp. 300-309, 1998.

[8] Marczak, R. J., Creus, G. J., Direct evaluation of singular integrals in boundary element analysis of thick plates, Engineering Analysis with Boundary Elements, 26, pp. 653-665, 2002.

[9] Gao, X.W. and Davies, T. G., Boundary Element Programming in Mechanics, Cambridge University Press, Cambridge, UK, 2002.

[10] Gao, X.W., The radial integration method for evaluation of domain integrals with boundary-only discretization. Engineering Analysis with Boundary Elements, 26, pp. 905-916, 2002. 
[11] Gao, X.W., A boundary element method without internal cells for twodimensional and three-dimensional elastoplastic problems, ASME Journal of Applied Mechanics, 69, pp. 154-160, 2002.

[12] Lachat, J.C. (1975) 'A Further Development of the Boundary Integral technique for Elastostatics', PhD thesis, University of Southampton, UK.

[13] Lachat, J.C. and Watson, J. O., Effective numerical treatment of boundary integral equation, Int. J. Numerical Methods in Engng, 10, pp. 991-1005, 1976. 